\title{
Management der schwergradigen Alveolarproteinose: Wie führt man sicher eine Ein-Lungen-Lavage durch?
}

\author{
Management of Severe Pulmonary Alveolar Proteinosis: How to Perform \\ Whole-Lung Lavage Safely
}

Autoren

Institut
R. Wittke', R. Wolf' ${ }^{1}$ T. Reinken², E. Müller², S. Ewig'

Thoraxzentrum Ruhrgebiet, Kliniken für Pneumologie und Infektiologie, Evangelisches Krankenhaus Herne und Augusta-Kranken-Anstalt Bochum

2 Thoraxzentrum Ruhrgebiet, Klinik für Anästhesiologie, Intensiv- und Notfallmedizin, Ev. Krankenhausgemeinschaft Herne/Castrop-Rauxel gGmbH eingereicht 30.1 .2011

akzeptiert $\quad 3.2 .2011$

Bibliografie

Dol http://dx.doi.org/

10.1055/s-0030-1256246

Online-Publikation: 24. 3. 2011

Pneumologie 2011; 65:

428-431 @ Georg Thieme

Verlag KG Stuttgart · New York ISSN 0934-8387

Korrespondenzadresse Prof. Dr. Santiago Ewig

Thoraxzentrum Ruhrgebiet Kliniken für Pneumologie und Infektiologie Evangelisches Krankenhaus Herne und Augusta-KrankenAnstalt Bochum Bergstraße 26

44791 Bochum

ewig@augusta-bochum.de

\section{Zusammenfassung}

$\nabla$

Die Alveolarproteinose ist eine seltene Erkrankung, gekennzeichnet durch eine Akkumulation von Lipoproteinen innerhalb der Alveolen. Bei einem 35-jährigen Patienten (Raucher und THCKonsum) diagnostizierten wir eine Alveolarproteinose mit schwerer respiratorischer (oxygenatorischer) Insuffizienz. Komplikativ bestand eine Pleuropneumonie. Die Therapie der Wahl besteht in einer therapeutischen Ein-Lungen-Lavage. Dieses Verfahren wird detailliert geschildert.

\section{Einleitung}

$\checkmark$

Die Alveolarproteinose ist eine seltene Erkrankung (Inzidenz 1:1000000) bei Erwachsenen mittleren Alters. Es besteht eine männliche Dominanz (Geschlechterverhältnis männlich:weiblich = 2:1). Pathopysiologisch besteht eine alveoläre Akkumulation von Lipoproteinen (PAS-positiv, z.B. Surfactant). Die Auskleidung der Alveolen führt radiologisch zu dem typischen Bild eines „crazy-paving“ (Kachelmuster) ( $\boldsymbol{\bullet}$ Abb. $\mathbf{1})$ und klinisch zu einer respiratorischen (oxygenatorischen) Insuffizienz. Umfangreiche Übersichten zur Alveolarproteinose sind verfügbar [1-4]. Hier soll speziell das therapeutische Verfahren der Ein-Lungen-Lavage detailliert beschrieben werden.

\section{Fallbeschreibung}

$\nabla$

Ein 34-jähriger Patient wurde aufgrund langsam zunehmender Luftnot in eine externe Klinik stationär aufgenommen. Es bestand keine Komorbidität. Der Patient war Raucher von 20 Zigaretten über 20 Jahre. Zudem bestand ein häufiger inhalativer THC-Konsum. Im konventionellen Röntgen des Thorax imponierte eine symmetrische Infiltration beider Lungen mit Aussparung lediglich

\section{Abstract \\ $\nabla$}

Pulmonary alveolar proteinosis (PAP) is an uncommon respiratory disorder which is characterised by an intraalveolar accumulation of lipoproteins. A 35-year-old patient (smoker and THC consumption) presented with a PAP and severe respiratory (type I) failure. In addition, the patient had pleuropneumonia. Repeated whole-lung lavage (WLL) is the treatment of choice. The procedure is presented in detail.

der Lungenspitzen ( $\bullet$ Abb. 2). Die CT des Thorax zeigte ein typisches „crazy-paving“-Muster (Kachelmuster) in nahezu allen Lungenfeldern. Es bestand eine leichtgradige restriktive Ventilationsstörung, jedoch eine schwergradige Einschränkung des Transferfaktors (TLCO/VA 26\% des Solls). In der Blutgasanalyse bei Raumluft bestand eine schwergradige Oxygenierungsstörung unter $\mathrm{Hy}$ perventilation.

Die transbronchiale Biopsie des Mittellappens ergab zunächst keine aussagekräftige Diagnose. Die BALF des Mittellappens war milchig trüb mit massenhaften Nachweis von PAS-positivem Präzipitat und zahlreichen Alveolarmakrophagen mit braunem Pigment. Unter Verkennung der Diagnose erfolgte eine systemische Steroid-Therapie.

Einen Monat nach Therapieeinleitung stellte er sich notfallmäßig mit starker Dyspnoe und neu aufgetretenen linksthorakalen Schmerzen vor. In der CT des Thorax ( $\bullet$ Abb. 1) wurde ein deutlicher Progress des „crazy-paving“-Musters mit einem konsolidierenden Infiltrat linksseitig nachgewiesen. Komplikativ bestand somit eine Pleuropneumonie links ( $\bullet$ Abb. 1). Bei schwerer Oxygenierungsstörung (Sauerstoffmaske mit $101 \mathrm{O}_{2} / \mathrm{min}$ : $\mathrm{pH} 7,41, \mathrm{pCO}_{2} 43 \mathrm{mmHg}, \mathrm{pO}_{2} 51 \mathrm{mmHg}$ ) wurde der Patient in unser Thoraxzentrum verlegt. 


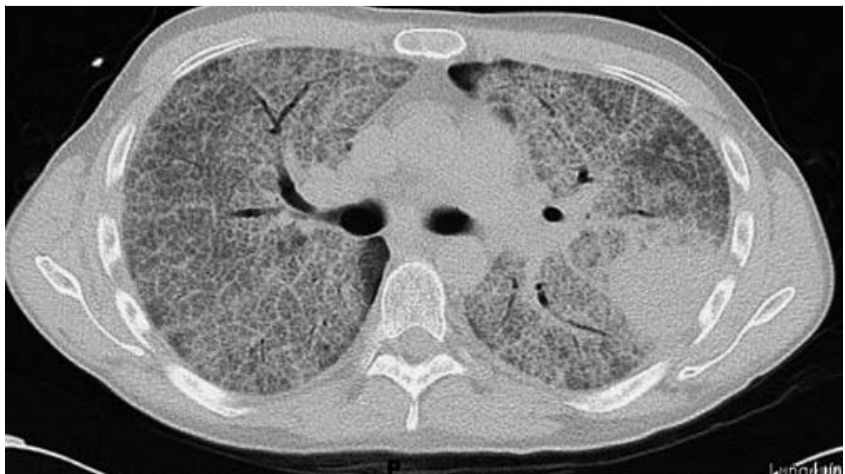

Abb. 1 „Crazy-paving“ (Kachelmuster) und Pleuropneumonie links.

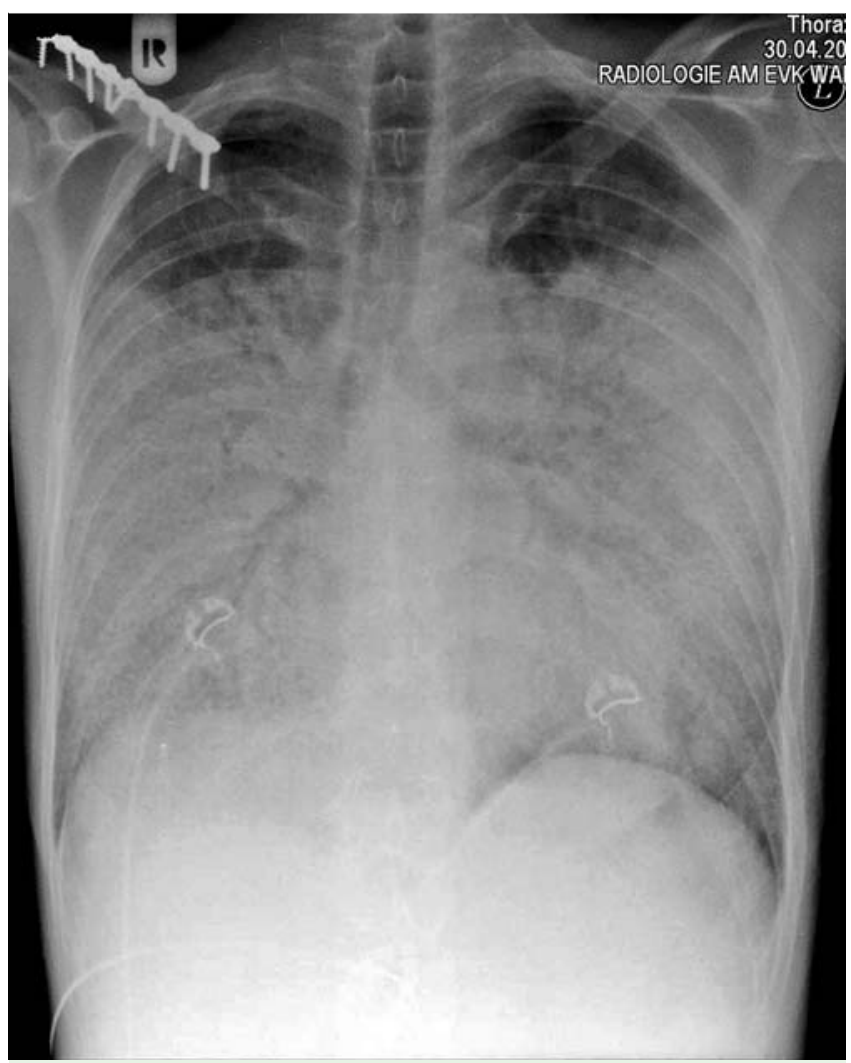

Abb. 2 Symmetrische Infiltration.

Bei CT-morphologisch typischem Aspekt einer Alveolarproteinose sowie PAS-Positivität in der BAL erfolgte die Diagnose einer PAP. Es erfolgte eine antimikrobielle Therapie sowie eine sequentielle therapeutische Ein-Lungen-Lavage in mehreren Sitzungen.

\section{Therapeutische Ein-Lungen-Lavage}

$\nabla$

Im Vorfeld der Ein-Lungen-Lavage wurden 401 Kochsalz (0,9\%) auf Körperwärme im Operationssaal temperiert.

Der Patient wurde aufgrund der bevorstehenden mehrstündigen Prozedur auf einem beweglichen Operationstisch mit einer Wärmematratze gelagert.

Nach Anlage eines periphervenösen Zuganges erfolgte die Einleitung der totalintravenösen Allgemeinanästhesie (Propofol, Ultiva) und Intubation mit einem Doppellumentubus (Größe 41 linksschenkelig) sowie die Anlage eines arteriellen, eines zentralven-

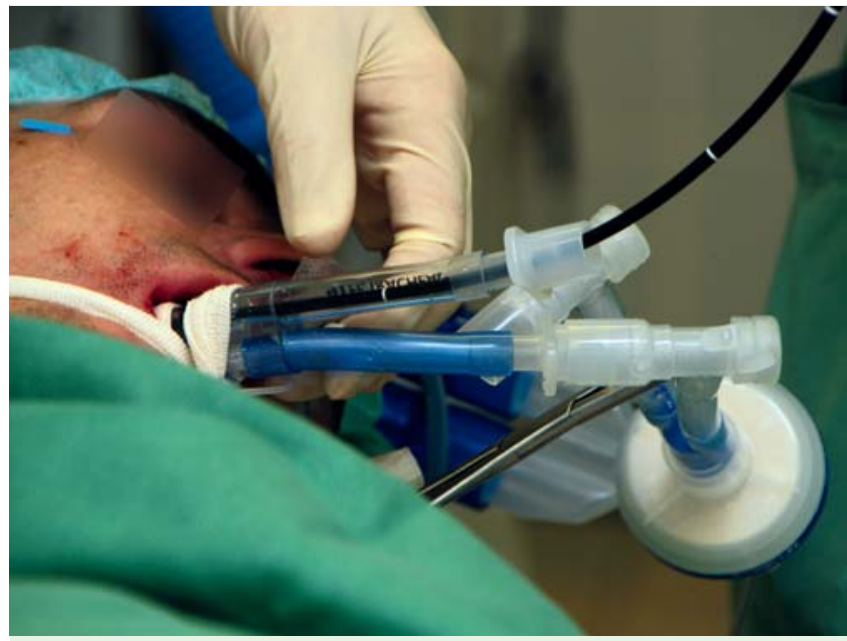

Abb. 3 Bronchoskopische Inspektion über den Doppellumentubus.

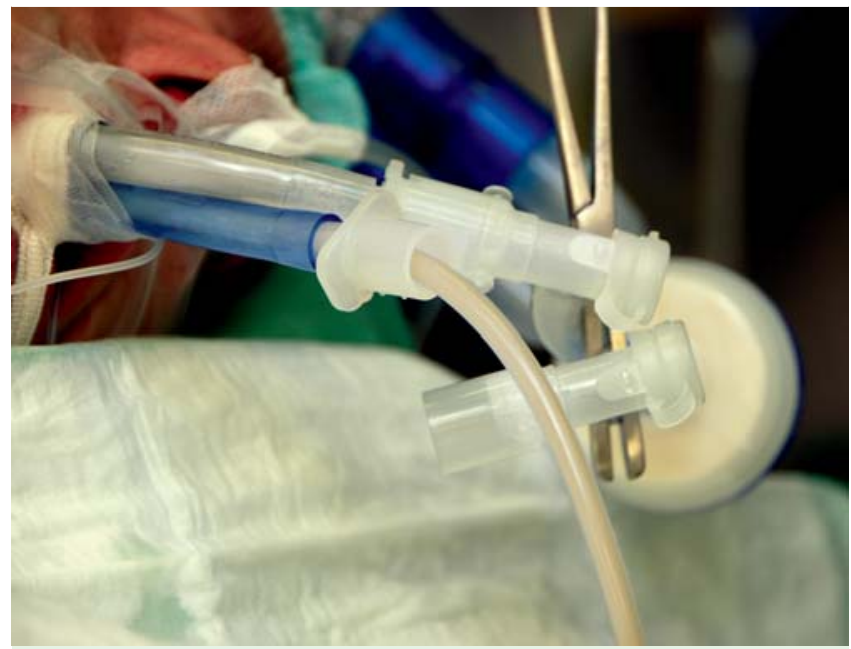

Abb. 4 Magensondenschlauch im bronchialen Schenkel des Doppellumentubus.

ösen Zuganges und eines Blasenverweilkatheters mit Thermokatheter. Die niedrige Compliance der Lunge erforderte ein niedriges Tidalvolumen mit hoher Atemfrequenz. Die $\mathrm{FiO}_{2}$ betrug $100 \%$. Ein erweitertes anästhesiologisches Monitoring erfolgte. Zusätzlich zum Standard-Anästhesiemonitoring (Beatmungsparameter, expiratorischer $\mathrm{CO}_{2}$ und $\mathrm{SaO}_{2}$ ) erfolgten eine arterielle Blutdruckmessung sowie halbstündliche Blutgas- und Elektrolytkontrollen. Die Anästhesieseite wurde rechts des Patienten gewählt mit Kopflage des Patienten in annähernd $90^{\circ}$ zur linken Seite ( Abb. 3). Links des Patienten wurde der Endoskopieturm sowie der Spül-Turm aufgebaut. Am Fußende des Patienten befand sich ein Monitor für die Bronchoskopie.

Zunächst wurde beidseitig bronchoskopisch via Doppellumentubus mit dem Babyscope der Tracheobronchialbaum inspiziert ( Abb. 3). Zu beachten ist die korrekte Lage des Cuffs im Hauptbronchus.

Über den bronchialen Schenkel des Doppellumentubus wurde mithilfe von Silikonspray ein Magensondenschlauch bis zum Ende des Bronchialschenkels eingeführt ( $\bullet$ Abb.4). Dieser Schlauch wurde durch ein Ypsilonstück mit einem auffüllbaren 2-l-Beutel für die Kochsalzzufuhr nach apikal verbunden. Der zweite Teil des Ypsilonstücks dient dem Ablauf nach basal in einen 2-1-Urinbeutel. Über dieses Schlauchsystem erfolgte die 


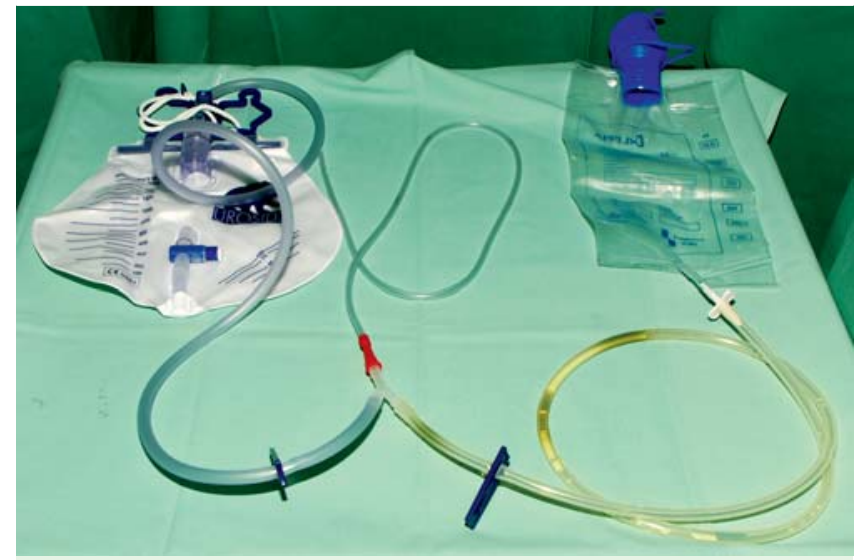

Abb. 5 Von links nach rechts: 2-I-Beutel - Einlaufschenkel - Y-Stück Magensonde - Ablaufschenkel - 2--Irinbeutel.

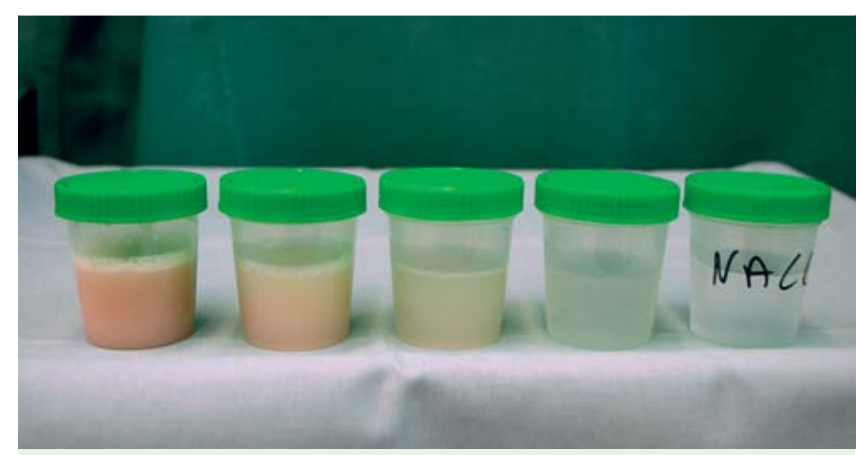

Abb. 6 Titrationsdarstellung der BALF.

therapeutische Lavage. Der Zu- und Ablauf wurde über zwei Klammern am jeweiligen Schenkel gesteuert ( $\bullet$ Abb. 5).

Die Ein- und Ausfuhr wurde bilanziert. Es ergab sich nach ca. 351 Instillation eine zu vernachlässigende Differenz von 200 - $300 \mathrm{ml}$ zugunsten der Einfuhr.

In unserem Fall bedeutete dies pro Sitzung insgesamt 351 Kochsalz portionsweise $(0,5-0,751)$. Die Portionsgröße richtete sich nach dem Füllstand der Lunge. Kurz vor Überlaufen (sichtbar im Tubusschenkel) wurde die Einlaufportion beendet. Der Zulauf erfolgte gesteuert durch den beweglichen Operationstisch in $30^{\circ}$-Kopfhochlage, der Ablauf in $30^{\circ}$-Kopftieflage. Eine Schräglage in Ablaufposition erbrachte keinen verbesserten Ablauf. Der 2-1-Beutel wurde jeweils aufgefüllt, der Urinbeutel bei Erreichen der 21 abgelassen und zur Titrationsdarstellung wurden jeweils $50 \mathrm{ml}$ in einem Urinbecher zur Seite gestellt ( $\bullet$ Abb. 6).

Initial war die Lavage ockerfarben mit viel Sediment. Die Lavage wurde beendet bei einem annähernd klaren Rücklauf der Lavage $(\circlearrowleft$ Abb.6), erwünschtes Ziel war eine klare Lavageflüssigkeit. Nach einer dreieinhalbstündigen Prozedur wurde die Untersuchung beendet und der Patient konnte wenige Minuten später extubiert werden, wurde jedoch weiterhin auf der Intensivstation überwacht.

Während der Lungenlavage kam es zu einer schwergradigen gemischt respiratorisch-metabolischen Azidose $\left(\mathrm{pH} 7,15, \mathrm{pCO}_{2}\right.$ $62 \mathrm{mmHg}, \mathrm{pO}_{2} 67 \mathrm{mmHg}, \mathrm{BE}-6,7 \mathrm{mmol} / \mathrm{l}$, Bikarbonat $17,7 \mathrm{mmol} /$ 1). Zum Zeitpunkt der Extubation hatte sich diese bereits schon deutlich gebessert und am Folgetag unter Sauerstoffzufuhr und Spontanatmung normalisiert.

Mögliche Komplikationen wie Hypernatriämie, Tubusdislokation, Pleuraerguss oder Pneumothorax traten nicht auf.

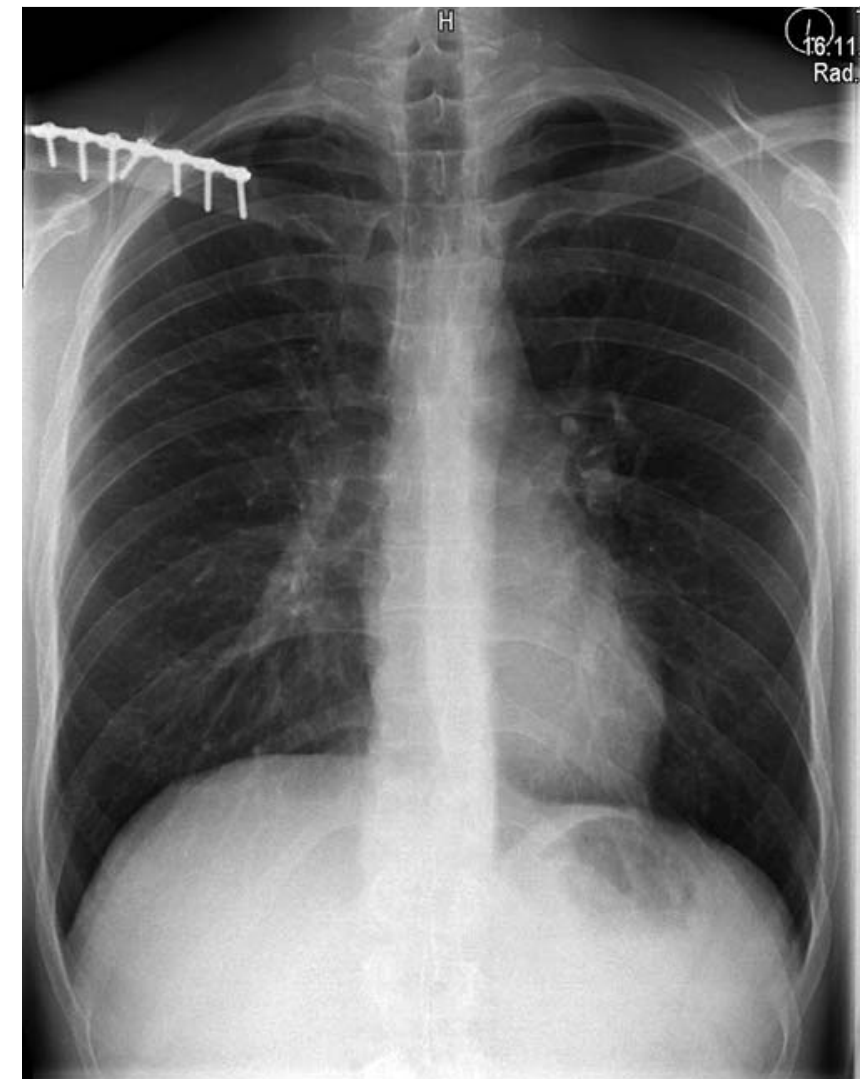

Abb. 7 Therapieerfolg nach 6 Monaten.

Drei Tage später erfolgte die linksseitige Ein-Lungen-Lavage nach demselben Prinzip.

\section{Verlauf}

$\nabla$

Die zweizeitige linksseitige Ein-Lungen-Lavage führte zu einem makroskopisch annähernd klaren Rücklauf der Lavage. Korrespondierend hierzu zeigte sich konventionell-radiologisch eine Befundbesserung sowie ein rückläufiger Sauerstoffbedarf. Es persistierte die nur leicht gebesserte schwergradige Einschränkung des Transferkoeffizienten (TLCO/VA 37\% des Solls). Die linksthorakalen atem- und bewegungsabhängigen Schmerzen wurden als Begleitpleuritis gedeutet und konnten analgetisch behandelt werden. Die Pneumonie wurde ohne Erregernachweis in der BALF kalkuliert antimikrobiell erfolgreich behandelt. Der Patient wurde zunächst mit einer Sauerstoffheimtherapie entlassen.

Der Patient stellte sich nach drei Wochen ambulant zur Verlaufskontrolle vor. Er beendete nachweislich das Rauchen. Es bestand zwar eine subjektive, lungenfunktionelle sowie CT-morphologische Besserung, bei ausbleibender Normalisierung der Befunde und unverändert schwergradiger Einschränkung des Transferkoeffizienten (TLCO/VA 36\% des Solls) mit mittelgradiger restriktiver Ventilationsstörung und ausgeprägter respiratorischer Partialinsuffizienz $\left(21 \mathrm{O}_{2} / \mathrm{min}: \mathrm{pH} 7,42, \mathrm{pCO}_{2} 39 \mathrm{mmHg}, \mathrm{pO}_{2}\right.$ $40 \mathrm{mmHg}$ ) wurde ein erneuter stationärer Termin mit dem Patienten zur erneuten therapeutischen Lavage vereinbart.

Es erfolgte dasselbe Prozedere wie im vorangegangenen stationären Aufenthalt. Nach komplikationsloser bilateraler Ein-LungenLavage bestand nur noch eine leichtgradige restriktive Ventila- 
tionsstörung und eine mittelgradige Reduktion des Transferkoeffizienten (TLCO/VA 50\% des Solls). Blutgasanalytisch wurde eine leichtgradige, nicht mehr sauerstoffpflichtige respiratorische Partialinsuffizienz nachgewiesen (bei Raumluft: $\mathrm{pH} 7,41, \mathrm{pCO}_{2}$ $39 \mathrm{mmHg}, \mathrm{pO}_{2} 61 \mathrm{mmHg}$ ).

Die weitere Verlaufskontrolle nahm der Patient erst 5 Monate später wahr. Es bestand eine klinische und konventionell radiologische Befundnormalisierung ( $\triangle$ Abb. 7). Lungenfunktionell bestand ein Normalbefund bei weiter steigendem Transferkoeffizienten (TLCO/VA 63\% des Solls). Die Blutgasanalysen waren sowohl in Ruhe als auch unter Belastung normwertig.

\section{Zusammenfassung}

$\nabla$

Die therapeutische Ein-Lungen-Lavage ist unverändert die Therapie der Wahl bei PAP. In Abhängigkeit vom Ausmaß der Alveolarproteinose sind wiederholte therapeutische Lavagen notwendig. Aufgrund der Seltenheit der Erkrankung wird die Ein-Lungen-Lavage jedoch nur selten durchgeführt. Es erfordert eine hohe pneumologisch-anästhesiologische Kompetenz und geht mit hohem zeitlichen und personellen Aufwand einher. Es sollte deshalb nur in Zentren mit entsprechender Erfahrung angewendet werden.

\section{Danksagung}

Wir danken für die kompetente Unterstützung durch Dr. M. Wiebel, Thoraxklinik Heidelberg.

\section{Interessenkonflikt}

Die Autoren geben an, dass kein Interessenkonflikt besteht.

\section{Literatur}

1 Trapnell BC, Whitsett JA, Nakata K. Mechanisms of Disease Pulmonary Alveolar Proteinosis. N Eng J Med 2003; 349: 2527-2539

2 Seymour JF, Presneil JJ. Pulmonary Alveolar Proteinosis Progress in the First 44 Years. Am J Respir Crit Care Med 2002; 166: 215 - 235

3 Kitamura T, Uchida K, Tanaka $N$ et al. Serological Diagnosis of Idiopathic Pulmonary Alveolar Proteinosis. Am J Respir Crit Care Med 2000; 162: $658-662$

4 Michaud G, Reddy C, Ernst A. Whole-Lung Lavage for Pulmonary Alveolar Proteinosis. Chest 2009; 136: 1678-1681 